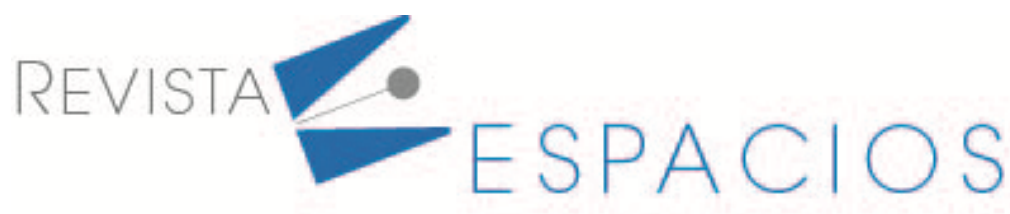

Vol. 41 (47) $2020 \cdot$ Art. 7

Recibido/Received: 01/07/2020 • Aprobado/Approved: 08/11/2020 • Publicado/Published: 10/12/2020

\title{
La gestión de los residuos sólidos en la ciudad de Barranquilla, Colombia
}

\section{Solid waste management in the city of Barranquilla, Colombia}

\author{
HERNÁNDEZ, Hugo G. ${ }^{1}$ \\ NIEBLES, William A. ${ }^{2}$ \\ FERIA, Jhon J. ${ }^{3}$
}

\begin{abstract}
Resumen
Son varios los aspectos que se revisan en cuanto a la gestión de residuos en la ciudad de Barranquilla, Colombia, pues los municipios han tenido variados inconvenientes en ello. En relación con lo anterior, mediante el uso de una metodología expositiva, se observaron las disposiciones vigentes y se examinó si las mismas resultaban pertinentes para estudiar la gestión de los residuos sólidos en la ciudad de Barranquilla. Finalmente, se plantea la idea de cómo la gestión de residuos es un reto para las localidades pues es un aspecto que aún está en construcción.
\end{abstract}

Palabras clave: desechos peligrosos, legislación ambiental, medio ambiente, PGIRS.

\begin{abstract}
There are several aspects that are reviewed in terms of waste management, as the municipalities have had various drawbacks in this. In relation to the above, through the use of an exposition methodology, the current provisions were observed, and it was examined whether they were pertinent to study solid waste management in the city of Barranquilla. Finally, the idea is raised of how waste management is a challenge for localities, insofar as it is an aspect that is still under construction.

keywords: hazardous waste, environmental legislation, environment, PGIRS.
\end{abstract}

\section{Introducción}

Las dinámicas económicas globales han cambiado la forma en que interactúan todos los sistemas integrados a la productividad (Acuña, 2001); este aspecto no es ajeno para la gestión ambiental y, por ende, todos los factores relacionados con este principio se encuentran hoy sometidos a regulaciones, observaciones y seguimientos, donde tanto los Estados como las organizaciones de gestión y promoción, lideran a nivel mundial (Álvarez, 2009).

Cada vez son más evidentes las afectaciones al medio ambiente ocasionadas por los cambios en los estilos de vida de la población mundial, y en especial en Colombia (Marshall \& Farahbakhsh, 2013); es así, como se reconocen aspectos como el uso y la tenencia de la tierra, de los cuales gran parte del territorio es destinado a

\footnotetext{
${ }^{1}$ Magister en Sistema de Gestión, Especialista en Estudios Pedagógicos, Especialista en Diseño y Evaluación de proyectos, Ingeniero Industrial. Docente Programa de Administración de Empresas Universidad del Atlántico-Colombia. hugoghernandezpalma@gmail.com

${ }^{2}$ Doctor en Ciencias Gerenciales, Magíster en Dirección Estratégica, Administrador de Empresas. Docente de la Universidad de Sucre. william.niebles@unisucre.edu.co

${ }^{3}$ Docente investigador. Facultad de Ingeniería. Universidad de Sucre, Colombia. jhon.feria@uniscure.edu.co
} 
las actividades de ganadería, generando fenómenos erosivos y otras afectaciones a la biodiversidad. Del mismo modo, se consideran los cambios en la dinámica demográfica, motivados por los grandes desplazamientos humanos del campo a las ciudades; y la propia cultura ambiental, que aún se encuentra en mora de su verdadera concientización (Pérez \& Castro, 2012).

De acuerdo con lo anterior, para todos los sistemas productivos, el aspecto ambiental se ha convertido en un elemento esencial que debe ser atendido (Abarca, Maas \& Hogland, 2015), no solo desde la óptica del lucro, sino también desde la visión de la sostenibilidad, en la medida en que de la correcta gestión que se realice al respecto, se deriva el bienestar común de las comunidades o sociedades (Enviromental Protection Agency, 2003). En este sentido, se considera que la gestión ambiental, como parte del desarrollo sostenible, despliega una visión de conjunto, incorporando los campos de estudio desde la cultura, la ética, la economía, la ecología y la eficiencia (Ramírez, Paredes \& Guerrero, 2014).

Lo citado, demuestra que no solamente se refiere a un enfoque teórico de desarrollo, sino que puede tomarse como un modelo reconocido, se caracteriza por aportar esquemas o iniciativas de desarrollo o concebirse como un objetivo específico en las estrategias definida por los municipios para alcanzar competitividad (Cevallos, Campos \& Nevares, 2016). Colombia ha respondido a estas exigencias, y expresión de ello son los diversos esquemas normativos que ha desarrollado para entregar parámetros sobre la gestión a desplegar en el tema objeto de estudio a todos los actores nacionales (Medina \& Cárdenas, 2010).

Al revisar el marco legislativo actual, se encuentra que los lineamientos esenciales parten de la Constitución Política, de 1991, donde se contempló el derecho de todos los individuos del territorio nacional, a contar con un medio ambiente sano, limpio, donde la gestión del Estado y demás integrantes del aparato productivo y económico nacional fuera relevante para preservar las condiciones de este (Ministerio de Ambiente, Vivienda y Desarrollo Territorial, 2015). A nivel internacional, son varios los acuerdos realizados; entre los más destacables se encuentran Los cinco acuerdos de Río, los cuales se han constituido en la respuesta más global e integral que busca establecer un régimen de cooperación internacional, donde el trabajo colaborativo abarque de forma integral todo lo referente al medio ambiente (Gardner, 2013).

En la declaración mencionada, se establece que en el crecimiento económico y social deben considerarse los fundamentos que hacen referencia a los procesos de producción, y observar cómo el consumo y otras actividades relacionadas pueden impactar en el bienestar común. Igualmente, hace una invitación a evaluar e insertar en los planes de gestión los lineamientos de responsabilidades comunes, aunque diferenciadas, denominados el Concepto de Estocolmo, como un mecanismo para la orientación de los programas y políticas en este aspecto (Gardner, 2013).

Es necesario resaltar también la importancia del Convenio de Basilea, el cual fue establecido a raíz del descubrimiento de depósitos de desechos tóxicos en África provenientes del extranjero, y cuyos principales objetivos van enmarcados en la responsabilidad que tiene cada uno de los países generadores sobre el manejo y disposición de sus propios desechos peligrosos (Rodríguez et al., 2014).

En este sentido, algunos estados han realizados esfuerzos para reglamentar la protección de los recursos naturales y subsidiariamente la salud de la población, por medio de políticas públicas y normas en las leyes, todo esto gracias a la preocupación internacional por la situación de contaminación que existe en el mundo (Rodríguez, García \& García, 2016), con esto se pretende hacer frente a la exigencia mundial, ajustando criterios y parámetros de vigilancia y seguimiento de los residuos, además, se busca la promoción de la sustentabilidad económica a partir del control de las acciones relacionadas con sustancias y residuos que afecten o coloquen en riesgo la vida humana (Muñoz et al., 2017).

En la actualidad, el país maneja consignas definidas para el manejo de los residuos o desechos peligrosos (RESPEL), tomando en cuenta todas esas sustancias, derivados o despojos, que cuentan con algún tipo de característica que afecte, influya o genere daños a la salud del individuo o al medio ambiente (Hernández \& Arango, 2013). De esta manera, en el Decreto 4741. de 2005, expedido por el Ministerio de Ambiente, Vivienda 
y Desarrollo Territorial (MAVDT), se presentan los criterios que dan pie a la clasificación de los distintos desechos de acuerdo con la actividad fuente o de origen.

Es así como la información sobre los RESPEL es registrada en el país por las autoridades ambientales bajo el Sistema de Información Ambiental del IDEAM (Instituto de Hidrología, Meteorología y Estudios Ambientales), los cuales son los encargados de administrar este aplicativo. Es decir, por medio de esta plataforma se logran las métricas estadísticas a fines con la producción, clasificación y actividades de los residuos en cuanto a su peligrosidad para el país. En el año 2010, por ejemplo, en Colombia se obtuvieron 138.090 toneladas de RESPEL, de los cuales, pocos fueron destinados a la prevención y minimización de la generación, y la mayoría se orientó al tratamiento de estos recursos para su posterior disposición final (Cubillos et al., 2015).

Bajo los principios enunciados en caso de la ciudad de Barranquilla se vienen gestionando los aspectos asociados a nivel nacional, y es a partir de este punto de partida que se realiza, especialmente en la ciudad de Barranquilla; seguidamente, un análisis de la situación actual, tomando como base los componentes vigentes, afectaciones al entorno y ventajas desarrolladas, según la información de referencia consultada como parte de esta investigación. El objeto final fue, entonces, extraer las reflexiones del caso que contribuyan con la profundización de estos conceptos y generar recomendaciones o sugerencias para el abordaje de esta temática en la ciudad de Barranquilla.

\section{Metodología}

Para el presente documento -y observando los postulados de autores como Arias (2012)- se realizó una revisión de literatura, con base en la utilización de una metodología que une técnicas cualitativas y descriptivas, que a su vez facilitan la recolección de la información a partir del análisis de aspectos característicos pretendidos en el estudio, con el fin de delinear los aspectos más relevantes frente al tema objeto de análisis y sus conceptos inherentes(Lambert \& Lambert, 2012; Taylor, Bogdan \& DeVault, 2015). Esta revisión de literatura se procesa por medio del análisis de contenido, proceso que permite extraer la información en categorías de manera que la misma responda a los elementos del estudio relacionados con la gestión integral de residuos sólidos en Colombia.

Esta tipología de investigación permite abordar dichos elementos sin intervenciones o manipulaciones por su corte descriptivo brindando así mismo la opción de conocer avances y hallazgos de otros investigadores en la misma línea de acción a la planteada en los objetivos de este documento (Luna et al., 2014). Dicho proceso se realiza de manera focalizada en la Ciudad de Barranquilla, ubicada en la Region Caribe colombiana.

\section{Resultados}

\subsection{Revisión bibliográfica: La gestión integral de residuos sólidos en Colombia}

De acuerdo con los propósitos globales actuales, la gestión ambiental se convierte en una herramienta fundamental para buscar mitigar el impacto negativo derivado del crecimiento acelerado de la población, los esquemas productivos modernos, y los niveles de consumo (Pires, Martinho \& Chang, 2011); es así como son requeridas metodologías que brinden los lineamientos para el establecimiento de objetivos y metas ambientales, así como también los procesos de planeación, organización y control de los resultados (Avellaneda, 2013). En este sentido, Forero (2013) propone el siguiente esquema de gestión ambiental. 
Figura 1

Esquema de la gestión ambiental sostenible

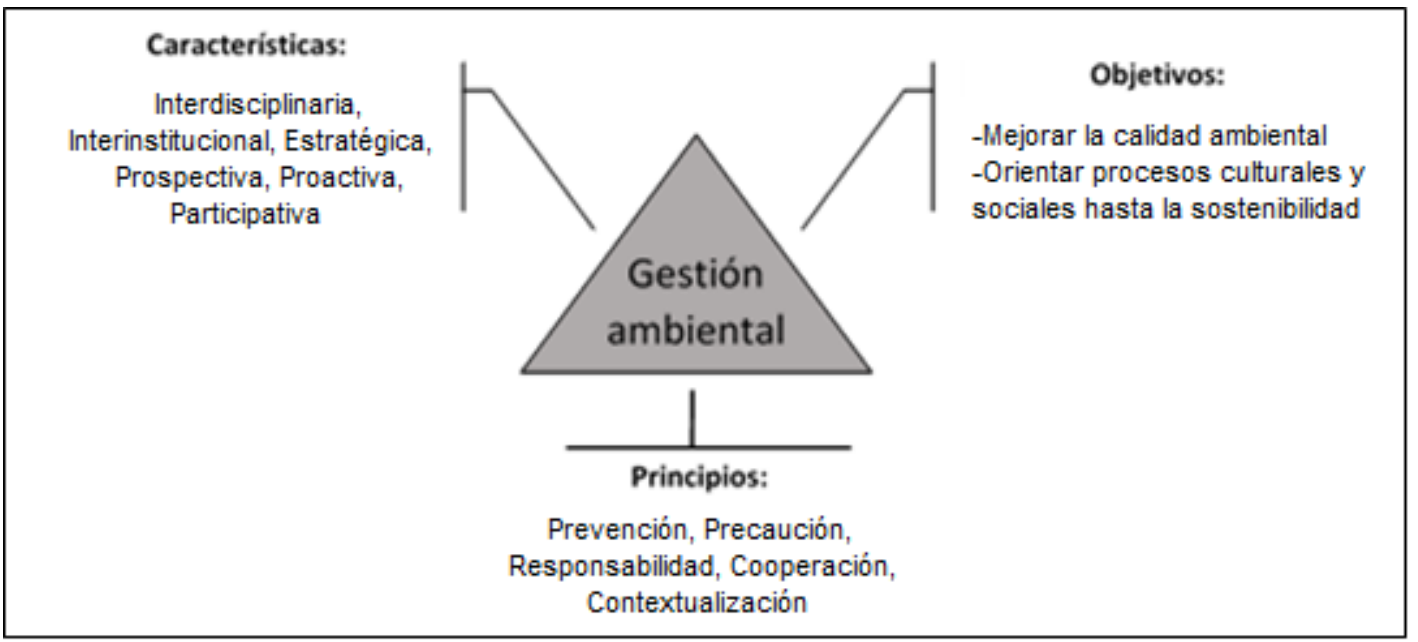

Fuente: Adaptado de Forero (2013)

Ahora bien, vale la pena resaltar que el manejo de los residuos genera un impacto importante en la salud de la población, por la transmisión de enfermedades por bacterias y parásitos, en la medida en que su presencia genera el contagio de agentes patógenos que pueden derivar en consecuencias graves (Laurent et al., 2014); del mismo modo, se produce la afectación por lesiones e infecciones que pueden ser originadas por el contacto con objetos corto punzantes que pudieran estar ubicados en los desechos sólidos; y finalmente, por la contaminación generada por la quema de los residuos, que genera afectaciones al sistema respiratorio de los habitantes cercanos a dichas disposiciones (Sáez \& Urdaneta, 2014).

Adicional a los efectos en el ser humano, la disposición inadecuada de residuos genera efectos en el medio ambiente en la medida en que genera contaminaciones en la tierra, el agua y el aire, las cuales pueden ser expandidas por los fenómenos de las lluvias y las aguas superficiales (Guerrero, Maas \& Hogland, 2013). Es así como un desconocimiento de los aspectos ambientales asociados con la disposición de los residuos sólidos puede generar un desmejoramiento en la calidad de vida de los habitantes de una región o país, impactando negativamente en su productividad, e incurriendo en elevados costos económicos y sociales (White, Dranke \& Hindle, 2012).

Tomando en cuenta lo anterior, y de acuerdo con las directrices entregadas por el marco normativo de Colombia, los Planes de Gestión Integral de Residuos Sólidos (PGIRS) se regulan en esencia por la Resolución 754, de 2014; sin embargo, existen otras leyes, decretos y resoluciones vigentes que pueden ser consideradas según cada caso y gestión en el territorio nacional (Ministerio de Vivienda y Desarrollo Territorial, 2014).

La resolución citada, articula cada una de las fases o etapas que se deben cumplir para la puesta en marcha de los PGIRS a nivel municipal, y es la disposición que actualmente viene aplicando el municipio de Barranquilla. En consecuencia, a continuación, se relacionan los componentes requeridos para la gestión de residuos, haciendo referencia a las leyes asociadas que la Resolución 754 ha puesto en consideración, y que son parte integral el PGIRS de esta localidad de Barranquilla:

- Residuos Aprovechables y No Aprovechables: se determina que los residuos deben clasificarse, y reciclar aquellos que por su origen, composición y estado residual puedan ser recuperados, así como también, separar aquellos que no cumplen con esta condición. A su vez, los residuos pueden dividirse en Ordinarios y Especiales; los Ordinarios se caracterizan por no agregar peligro, y usualmente provienen de actividades como barrido, aseo y limpieza en general; por otra parte, los Especiales son aquellos que necesitan de un proceso más estructurado para su manejo, y dentro de ellos, se puede ubicar otra escala 
haciendo referencia a los de Construcción, que habitualmente son los derivados de las demoliciones o actividades conexas a obras civiles (Ministerio de Vivienda y Desarrollo Territorial, 2014).

- Gestión de los actores o integrantes: los municipios están obligados a planear, divulgar y ejecutar los PGIRS, como mecanismo de seguimiento y control de los residuos generados. Igualmente, deben velar por implementar las medidas y mejoras necesarias para que los demás responsables o generadores apropien las medidas estimadas (Mejía, 2010).

- Los actores involucrados actualmente en el municipio de Barranquilla son: Empresa de Servicios Públicos, recicladores formalizados y generadores residenciales y no residenciales (comercio, industria y demás sectores productivos). Lo enunciado se ilustra a continuación en la fig. 2.

Figura 2

Estructura general del PGIRS

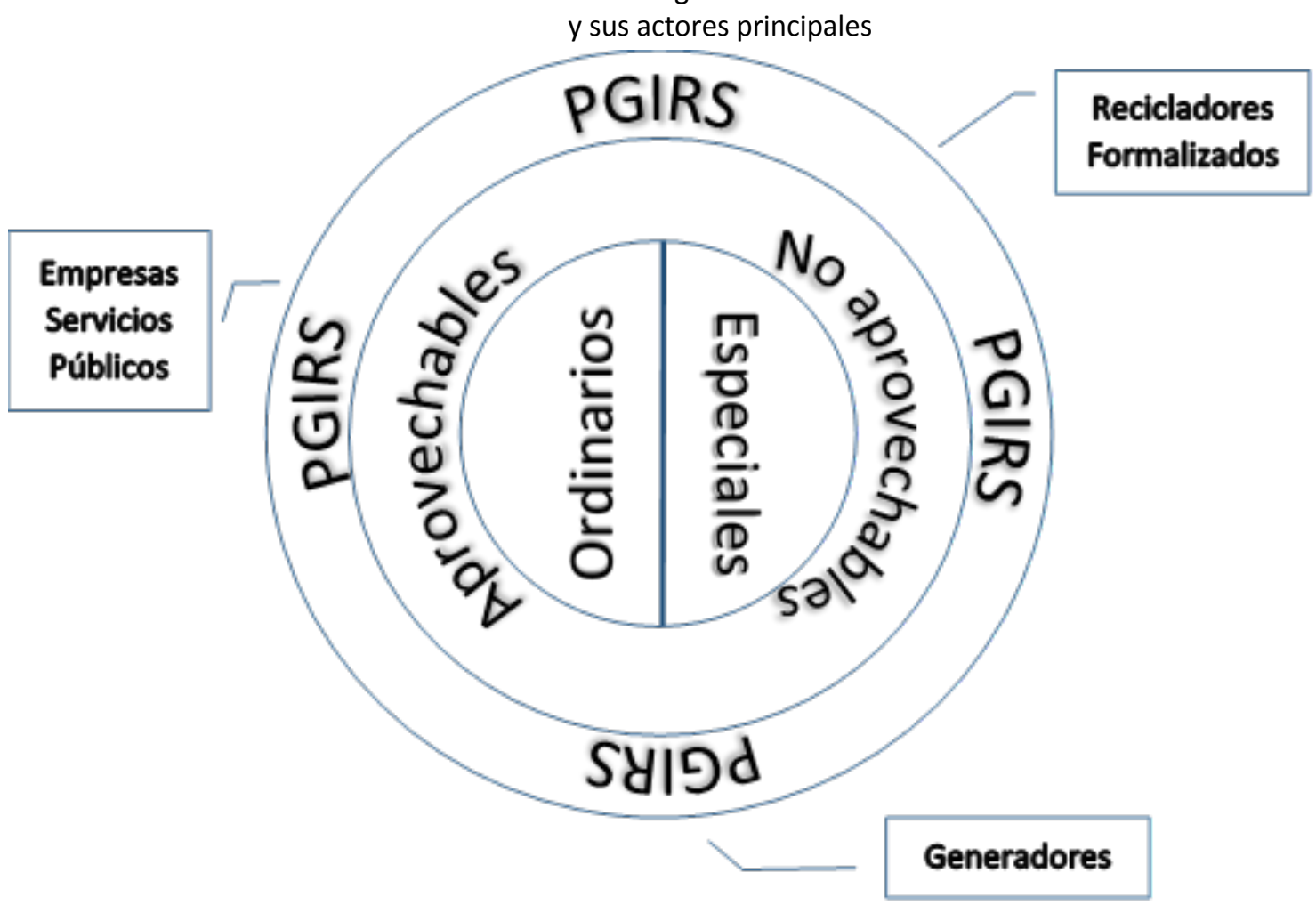

Fuente: Los Autores

Lo expresado contempla, además:

- Parámetros de Base: estos incluyen todas las actuaciones y disposiciones que deben cumplirse, tanto por los responsables como por los demás vinculados a los PGIRS, y triangula los alcances de uno y otros para lograr la gestión oportuna según cada requerimiento. Es así como se pueden relacionar los distintos componentes involucrados, que son presentados en la tabla 1. 
Tabla 1

Componentes del PGIRS

\begin{tabular}{l} 
Componentes del PGIRS \\
\hline Esquema de prestación de servicios por cada actividad \\
\hline Prestadores del servicio público en el municipio \\
\hline Tarifas aplicables para la prestación de servicios \\
\hline Proyección de la cantidad de residuos generados por \\
sector, manejo y disposición final de los mismos \\
\hline Cobertura de la prestación de servicios a nivel municipal \\
\hline Frecuencia de recolección de los residuos \\
\hline Puntos críticos de generación \\
\hline Manejo especial para áreas naturales y comunes, como \\
playas o similares \\
\hline Corte de césped y poda de árboles \\
\hline Inventario de puentes y vías públicas de atención \\
\hline Identificación de zonas industriales y de construcción \\
\hline Inventario de recicladores formales \\
\hline Sitio y manejo para la disposición final \\
\hline Acuerdos territoriales y condiciones del entorno \\
\hline Fuente: Adaptado de Ministerio de Vivienda \\
y Desarrollo Territorial (2014)
\end{tabular}

De acuerdo con lo expresado anteriormente, el PGIRS desarrollado en un contexto municipal de Barranquilla contribuye a la armonización de los planes del municipio, a la vez que reduce los impactos ambientales, los costos de consultoría, de control y seguimiento que deben incurrirse por parte de las autoridades ambientales (Diazgranados, Camargo \& Brochel, 2011).

Ello se debe a que este proceso brinda la construcción de un proceso de trabajo organizado para el desarrollo de una visión amplia sobre todos los elementos que puedan tener alguna incidencia en la gestión de residuos y así elaborar un proceso de planificación municipal basándose en los hallazgos.

\subsection{Impactos negativos 0 aspectos para reestructurar en el PGIRS del municipio de Barranquilla}

Los impactos negativos que, según la alcaldía de Barranquilla, vienen siendo objeto de intervención, están relacionados con el seguimiento y actualización de los programas para el fomento de la reducción, manejo, separación en la fuente, recogida selectiva y aprovechamiento de los residuos sólidos (Universidad del Norte, 2015).

Según la fuente citada, aunque los niveles de cobertura han mejorado notoriamente en los últimos años, existen aspectos susceptibles de evaluación, como la recolección, manejo y disposición de basuras. Al día de hoy, la disposición final se realiza en rellenos sanitarios, que pueden estar generando impactos negativos porque aún no se ha evolucionado en el tratamiento y enfoque de sostenibilidad que permita insertar a los Planes de Gestión Ambiental Regional (PGAR) los comandos o parámetros mejorados (López, 2011; Álvarez, 2016).

Otro aspecto que debe examinarse son las frecuencias de recogida, pues, aunque las empresas responsables han establecido un programa ajustado a las necesidades básicas de cada localidad, algunas han tenido variaciones notorias por el aumento de la actividad comercial, industrial y de servicios, lo que demanda en este momento una reprogramación, para lograr la cobertura optima que posibilite una gestión más oportuna, que contemple 
de manera adicional las variaciones o cambios que el crecimiento socio-económico va generando para el municipio (Álvarez, 2009; Universidad del Norte, 2015).

\subsection{Aspectos positivos de los PGIRS municipales en la ciudad de Barranquilla}

Como todo programa de control, manejo y seguimiento, los PGIRS del municipio de Barranquilla, viene siendo objeto de seguimiento por parte de diversos organismos, encontrando entre los aspectos que generan ventaja competitiva en la actualidad: la cobertura de recolección, manejo y disposición de los residuos generados en el municipio, la cual se estima en un $75 \%$, escala que se ha mantenido los últimos años desde la implementación del programa de recogidas semanales que atiende los sectores demarcados con una frecuencia de tres días, lo que a su vez viene arrojando un indicador bastante satisfactorio en cuanto al manejo de los desechos (Carmona \& Echavarría, 2015).

Adicionalmente, los residuos en las zonas urbanas, si bien han crecido, también vienen siendo gestionados de manera apropiada, aunque los estudios señalados muestran una gran oportunidad de mejora en los aspectos de sostenibilidad, por lo cual, se proyecta que este aspecto puede posicionarse aún más en los años siguientes y optimizar la gestión enriqueciendo los programas actuales con los avances que las concepciones de sostenibilidad vienen incorporando a la gestión medioambiental (Noguera \& Olivero, 2010).

Finalmente, otro aspecto a resaltar es la generación de cultura que los PGIRS han derivado en la comunidad, ya que en años anteriores se habían logrado algunos avances, pero ninguno tan notorio como los observados actualmente, y aunque todavía existen aspectos a mejorar si es destacable el cambio de manejo, control y disposición que se viene dando por parte de todos los actores asociados al proceso integral (Perdomo \& Ramírez, 2010; Problemática Ambiental, 2016; Gavidia, 2018).

\section{Conclusiones}

Al observar los parámetros generales que componen los PGIRS en Barranquilla se puede enunciar que los mismos se fundamentan en la normativa vigente y se han tomado en cuenta las directrices para tal fin; no obstante, este tipo de programas deben ser actualizados, valorados y renovados de manera constante, ajustando el marco legal a los requerimientos que el crecimiento mismo de la comunidad exige. Cabe resaltar que en la ciudad de Barranquilla viene creciendo en actividades de corte industrial y del sector de la construcción lo que ha incorporado al panorama de desarrollo una mayor cantidad de residuos.

En este mismo sentido, la necesidad de insertar aspectos como la sostenibilidad para potenciar el alcance de los PGIRS, deben ser analizados con miras a optimizar la gestión medioambiental, factor esencial para el desarrollo del aparato socio-económico de las localidades. A partir de lo anterior, las recomendaciones a realizar corresponden a las siguientes:

- Conformar equipos interdisciplinarios donde se vinculen personas que cuenten con la formación académica requerida para abordar las renovaciones o actualizaciones de los PGIRS y, a partir del trabajo idóneo, lograr las innovaciones necesarias para el buen uso de los recursos dispuestos por el municipio de Barraquilla, articulando la participación de todos los actores.

- Los actores directamente implicados serían las alcaldías municipales, la gobernación y los ministerios de interés; desde estos organismos se deben desarrollar las actuaciones necesarias para promover una gestión medio ambiental ajustada a las tendencias observadas.

- Estas iniciativas se proponen para periodos de ejercicio anual, pues los movimientos económicos y la organización social cambian de manera permanente, lo que demanda una revisión adecuada de los programas de gestión que implican el bienestar común. De este modo, es fundamental la puesta en 
marcha de procesos estandarizados hacia la gestión de los residuos sólidos en la ciudad de Barranquilla, con especial énfasis con la contaminación e impacto ambiental generado hacia el Rio Magdalena.

- La observancia de los avances mundiales puede ser un factor diferencial que propicie no solo los aportes al tema, sino también innovaciones oportunas para la mejora de la calidad de vida que beneficie las partes de interés, especialmente dentro de la ciudad de Barranquilla donde existe una importante necesidad por desarrollar procesos efectivos encaminados a la gestión de desechos sólidos de las diversas industrias existentes.

- Es indispensable que se pongan en marcha procesos a nivel industrial que garanticen que realmente se lleve a cabo una gestión de residuos sólidos correcto dentro de la ciudad de Barranquilla, pues en ocasiones desde la industria se prefiere incurrir en las diversas multas y violaciones que realizar dichos procesos de manera correcta.

- La concientización hacia el sector productivo resulta ser muchas veces el factor diferencial dentro de los países en vías de desarrollo, donde muchas veces la falta de conocimientos trae como resultados que se tomen decisiones negativas desde los cargos directivos u operativos con relación a la gestión de residuos, en este caso los sólidos.

\section{Referencias bibliográficas}

Abarca, L. A., Maas, G., \& Hogland, W. (2013). Solid waste management challenges for cities in developing countries. Waste management, 33(1), 220-232. https://doi.org/10.1016/j.wasman.2012.09.008

Acuña, G. (2001). Gestión ambientalmente adecuada de residuos urbanos en América Latina: un enfoque de política integral. En: Desafíos e innovaciones en la gestión ambiental: actas del Seminario Internacional Experiencia Latinoamericana en Manejo Ambiental"-LC/L. 1548-P-2001-p. 51-58". Recuperado de https://repositorio.cepal.org/handle/11362/7072

Álvarez. V.A. (2016) Mientras crece basura, rellenos se acercan a su recta final. Diario El Colombiano. Recuperado de http://www.elcolombiano.com/antioquia/vida-util-de-rellenos-sanitarios-en-cuentaregresiva-FY4032869.

Álvarez-Monsalve, C. A. (2009). Gestión integral de residuos sólidos: Minimización, almacenamiento, manipulación, transporte, aprovechamiento, tratamiento y eliminación. Disposición Final. Manizales, Colombia: Universidad de Manizales.

Arias, F. G. (2012). El proyecto de investigación. Introducción a la metodología científica. 6ta. Caracas, Venezuela: Editorial Episte, C.A.

Avellaneda, A. (2013). Gestión ambiental y planificación del desarrollo: el sujeto ambiental como actor político. Bogotá, Colombia: Ecoe Ediciones.

Carmona, E. J., \& Echavarría, K. S. (2015). La generación de residuos sólidos domiciliarios y sus principales determinantes para el departamento de Santander 2007 - 2013 : un estudio de caso. Recuperado de https://ciencia.lasalle.edu.co/ economia/244

Cevallos, U., Campos, T., \& Nevares, G., (2016). Relación gestión ambiental-competitividad territorial bajo el enfoque de la prospectiva estratégica. Revista DELOS: Desarrollo Local Sostenible, 9(25), 1-12. Recuperado de https://dialnet.unirioja.es/servlet/articulo?codigo=6494733 
Cubillos, V., González, M. Y., Ruiz, S., Vélez, R., \& Paredes, C., (2015). Estrategias de producción más limpia para el adecuado manejo y reducción en el origen de residuos peligrosos: caso de estudio industrias litográficas y tintorerías. Scientia et technica, 20(4), 396-405. http://dx.doi.org/10.22517/23447214.9157

Diazgranados, N. L. Camargo, A. S. Brochel, N. A. V. (2011) Estrategia regional de gestión de residuos. Caso: Área Metropolitana de Barranquilla (Colombia)". En: Hacia la sustentabilidad: Los residuos sólidos como fuente de energía y materia prima, SIIR, pp. 181-186. Recuperado de http://www.redisa.net/doc/artSim2011/GestionYPoliticaAmbiental/Estrategia\%20regional\%20de\%20gesti \%C3\%B3n\%20de\%20residuos.\%20Caso_\%C3\%81rea\%20Metropolitana\%20de\%20Barranquilla_Colombia.p $\mathrm{df}$

Enviromental Protection Agency. (2003). Manejando sus residuos peligrosos. Una guía para empresas pequeñas. Washington D.C., USA: EPA.

Forero, M. P. (2013). Lineamientos para la implementación y seguimiento de los PGIRS. Estudio de caso cinco municipios de Cundinamarca (Tesis de Maestría). Pontificia Universidad Javeriana. Bogotá, Colombia. Recuperdo de https://repository.javeriana.edu.co/handle/10554/12375

Gardner, G. (2013). Municipal solid waste growing. In Vital Signs (pp. 88-90). Washington, D.C.: Island Press. . http://dx.doi.org/10.5822/978-1-61091-457-4_21

Guerrero, L. A., Maas, G., \& Hogland, W. (2013). Solid waste management challenges for cities in developing countries. Waste management, 33(1), 220-232. http://dx.doi.org/10.1016/j.wasman.2012.09.008

Gutberlet, J. (2015). More inclusive and cleaner cities with waste management co-production: Insights from participatory epistemologies and methods. Habitat International, 46, 234-243.

https://doi.org/10.1016/j.habitatint.2014.10.004

Hernández, V. R., \& Arango, J. A. (2013). Evolución de la normativa ambiental colombiana en función de las tendencias mundiales de desarrollo sostenible. Revista NOOS, 3(4). 34-55. Recuperado de https://www.researchgate.net/publication/281526157_EVOLUCION_DE_LA_NORMATIVA_AMBIENTAL_C OLOMBIANA_EN_FUNCION_DE_LAS_TENDENCIAS_MUNDIALES_DE_DESARROLLO_SOSTENIBLE

Lambert, V. A., \& Lambert, C. E. (2012). Qualitative descriptive research: An acceptable design. Pacific Rim International Journal of Nursing Research, 16(4), 255-256. Recuperado de https://he02.tcithaijo.org/index.php/PRIJNR/article/view/5805

Laurent, A., Bakas, I., Clavreul, J., Bernstad, A., Niero, M., Gentil, \& Christensen, T. H. (2014). Review of LCA studies of solid waste management systems-Part I: Lessons learned and perspectives. Waste management, 34(3), 573-588. https://doi.org/10.1016 / j.wasman.2013.10.045

López. I. (2011). Gestión Integral de Residuos Sólidos. Módulo didáctico. Palmira, Colombia: Universidad Nacional Abierta y a Distancia - UNAD. Recuperado de https://es.scribd.com/document/233251726/Modulo-Unad

Luna, E. G., Navas, D. F, Mayor, G. A, \& Buitrago, L. A. B. (2014). Metodología para la revisión bibliográfica y la gestión de información de temas científicos, a través de su estructuración y sistematización. Dyna, 81(184), 158-163. https://doi.org/10.15446/dyna.v81n184.37066

Marshall, R. E., \& Farahbakhsh, K. (2013). Systems approaches to integrated solid waste management in developing countries. Waste management, 33(4), 988-1003.

http://dx.doi.org/10.1016/j.wasman.2012.12.023 
Medina, P. A., \& Cárdenas, D. C. B. (2010). La sostenibilidad ambiental urbana en Colombia. Bitácora urbano territorial, 2(17), 73-93. Recuperado de https://revistas.unal.edu.co/index.php/bitacora/article/view/18893

Mejía, J. A. S. (2010). La política ambiental y su institucionalidad en Colombia. Medio Ambiente \& Derecho: Revista electrónica de derecho ambiental, (20), 7. Recuperado de https://huespedes.cica.es/gimadus/20/07_john_alexander_salinas_mejia.html

Ministerio de Vivienda y Desarrollo Territorial (MVDT). (2014). Resolución 754 de 2014, Recuperado de http://www.minvivienda.gov.co/Documents/ViceministerioAgua/PGIRS/PGIRS\%20de\%20Segunda\%20Gen eraci\%C3\%B3n/Gu\%C3\%ADa\%20para\%20la\%20formulaci\%C3\%B3n,\%20implementaci\%C3\%B3n,\%20evalu aci\%C3\%B3n,\%20seguimiento,\%20control\%20y\%20actualizaci\%C3\%B3n\%20de\%20los\%20PGIRS.pdf.

Muñoz, A. L., Franco, C. A., Triviño, H. F., \& Álvarez, R. (2017). Evaluación de la gestión de residuos peligrosos (RESPEL) y sus implicaciones en el desarrollo sostenible de las actividades productivas en cinco municipios del departamento del Quindío, Colombia. Revista Luna Azul, (44), 334-347. http://dx.doi.org/10.17151/luaz.2017.44.20.

Noguera, K., \& Olivero, J. (2010). Los rellenos sanitarios en Latinoamérica: caso colombiano. Revista de la Academia Colombiana de Ciencias Exactas, Físicas y Naturales, 34(132), 347-356. Recuperado de http://www.accefyn.com/revista/Vol_34/132/347-356.pdf

Perdomo, J. \& Ramírez, J. A. (2010). Estrategia sobre ubicación y funcionamiento de estaciones de transferencia para el manejo de residuos sólidos en Colombia. Documentos CEDE 007715. Universidad de los AndesCEDE. Recuperado de https://ideas.repec.org/p/col/000089/007715.html

Pérez, G. I. A., \& Castro, L. G. (2012). Degradación, pérdida y transformación de la biodiversidad continental en Colombia: Invitación a una interpretación socioecológica. Ambiente y Desarrollo, 16(30), 53-71. Recuperado de https://dialnet.unirioja.es/servlet/articulo?codigo=4021796

Pires, A., Martinho, G., \& Chang, N. B. (2011). Solid waste management in European countries: A review of systems analysis techniques. Journal of environmental management, 92(4), 1033-1050. https://doi.org/10.1016/ j.jenvman.2010.11.024

Problemática Ambiental. (2016) Problemática ambiental en municipios de Colombia. Recuperado de http://problemabiental.blogspot.com.co/

Ramírez, C. A., Paredes, D., \& Guerrero, J. (2014). Financial and economic sustainability of urban solid waste recovery facilities in Colombia. Ingeniería y competitividad, 16(2), 65-77. Recuperado de https://dialnet.unirioja.es/servlet/articulo?codigo $=6043696$

Rodríguez, R., González, V., Alvarado, M., \& Soto, C., (2014). Planes de manejo de residuos químicos peligrosos: revisión bibliográfica. Vidsupra, Visión Científica, 6(1). 27-32. Recuperado de https://www.ciidirdurango.ipn.mx/assets/files/ciidirdurango/docs/VIDSUPRA/REVISTAS/VSV6N1.pdf

Rodríguez, J. P., García, C. A., \& García, M. C. (2016). Environmental management in public hospitals: Environmental management in Colombia. Revista de la Facultad de Medicina, 64(4), 621-624. http://dx.doi.org/10.15446/revfacmed.v64n4.54772

Sáez, A., \& Urdaneta, J. A. (2014). Manejo de residuos sólidos en América Latina y el Caribe. Omnia, 20(3), 121135. Recuperado de https://www.redalyc.org/pdf/737/73737091009.pdf 
Taylor, S. J., Bogdan, R., \& DeVault, M. (2015). Introduction to qualitative research methods: A guidebook and resource. New Jersey, USA: John Wiley \& Sons.

Universidad del Norte. (2015) Un norte para Puerto Colombia. Lineamientos estratégicos y Plan de acción para la competitividad del municipio de Puerto Colombia. Recuperado de http://www.uninorte.edu.co/documents/5564742/9b4a8361-6967-4524-8687-927215fdb588.

Usaquén, M y Sánchez, M. (2012). Economía de los servicios públicos domiciliarios, más allá del mercado y del Estado. Equidad y Desarrollo, (17), 31-56. https://doi.org/10.19052/ed.66

Vargas J. (2015). Análisis de los determinantes en la generación de residuos sólidos en el departamento del Tolima (2007-2012) (Tesis de pregrado en Economía). Universidad de la Salle. Bogotá, Colombia. Recuperado de https://ciencia.lasalle.edu.co/economia/752

White, P., Dranke, M., \& Hindle, P. (2012). Integrated solid waste management: a lifecycle inventory. Ebrlin, Alemania: Springer Science \& Business Media, P.P 85.

Esta obra está bajo una Licencia Creative Commons Attribución-NoCommercial 4.0 International

(cc) BY-NC 